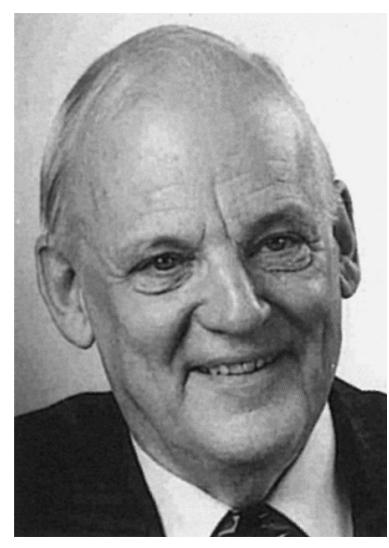

Gösta Samuelson gosta.samuelson@htu.se

\section{Global strategy on diet, physical activity and health}

A $\mathrm{n}$ expert report aiming to design strategies in promoting healthy diets and physical activity behaviours was published a year ago by the United Nations Food and Agriculture Organization (FAO) and the World Health Organization (WHO) (WHO Technical Report Series No. 916, 2003). The global strategy aims to combat constructively the increasing problem of chronic diseases, on all levels in countries around the world. Overweight, obesity, type 2 diabetes, cardiovascular diseases, including high blood pressure, and cancer account for more than $60 \%$ of all deaths worldwide and about half of the global burden of disease. In a special report in this issue, Amalia Waxman, from the WHO, discusses the policy aspects of the global strategy against the epidemic of these non-communicable diseases and the WHO's work so far.

\section{Key risk factors for chronic disease}

The most important risk factors are unhealthy diet and physical inactivity, as well as smoking. The sedentary lifestyle has been adopted in both developed and developing countries. People today are exposed to aggressive advertising in the mass media and an ample supply of inexpensive, energy-dense food, which is often high in sugar, fat and salt. Many intervention studies around the world have shown the benefits of health-promotion programmes focused on better dietary habits, increased physical activity and cessation of smoking. The newly adopted WHO global strategy is aimed at governments, the food industry, advertising bodies and health professionals, as well as towards individual counselling in healthy eating and physical activity. The scientific evidence is overwhelming that a change towards better dietary habits, increased physical activity and non-smoking is effective in reducing the risk factors. The strategy needs to be implemented urgently.

\section{Health promotion}

In the promotion of healthy eating habits an evaluation of partnerships between authorities, research groups and non-governmental organizations has been performed in Denmark. Bent Egberg Mikkelsen and Ellen Trolle show in their article that such partnerships are promising. It is, however, not easy to evaluate the effect because so many other factors operate at the same time. Moreover, nothing is known about the long-term effects.
The contribution by Lena A Nilsson, on Health promotion as school development, describes the school as an arena for health promotion. All school subjects might be considered effective learning cells for discussions of food habits and choice, as well as physical activity, which may result in deeper understanding and new practices.

\section{To fast or not to fast}

Recent very important studies in adults have shown that fasting before surgery should be avoided. Instead, an oral carbohydrate supply $2-3 \mathrm{~h}$ before an operation counteracts postoperative metabolic problems. Olle Ljungqvist emphasizes in his article that the treatment is simple and safe. In an invited commentary, the paediatric surgeon Staffan Meurling concludes that it is also likely that children should not fast for more than $2 \mathrm{~h}$ before elective surgery.

\section{Glycaemic index in food products}

A special report reviews the glycaemic index (GI). An internationally standardized method for GI determination in food is essential. Many aspects of GI are scrutinized, such as methodology, clinical aspects and practical implementation. Summarizing the present knowledge, GI should be used not in isolation, but together with other characteristics in evaluated food products. Although some studies indicate that low GI diets may favourably influence risk factors for chronic disease, more studies are needed to substantiate the significance of GI in healthy people. 